Case Study

\title{
Fault tolerance of reconfigurable pixel antenna using graph models
}

\author{
Raji George $^{1}$ - C. R. S. Kumar ${ }^{1}$ S. A. Gangal ${ }^{2} \cdot$ Makarand Joshi $^{3}$
}

(c) Springer Nature Switzerland AG 2019

\begin{abstract}
This paper discusses the use of graph models for fault tolerance of reconfigurable antennas. Reconfigurable antennas can adapt to their environment and change their operation depending on the user's request. Graph models are introduced for each configuration of the reconfigurable antenna to achieve the desired radiation performance. The use of graph models reduces the complexity while preserving its reliability allowing incorporations into applications like cognitive radio, MIMO, satellite communication and personal communication. A swifter response time is achieved with less cost and less losses. This method facilitates the development of the programming process for reconfigurable antennas. The graph theory facilitates the software control and also optimizes their performances. Graph modeling is also used to reduce the redundancy, complexity and increases the reliability of the reconfigurable antenna. The full analysis of these parameters allows a better implementation of the reconfigurable antenna in wireless and space communication. These models help the designers to analyze the antenna structures and transforms from bulky devices into mathematical and software models.
\end{abstract}

Keywords Reconfigurable ·Vertices · Edges · Graph · Fault tolerance

\section{Introduction}

Antennas were traditionally designed to operate on a single frequency. In applications like cognitive radio, it should access the full radio spectrum with rapid switching. Reconfigurable antenna is used as a solution to this. Reconfigurable antennas provide spectrum reuse, improve the unwanted signal gain or mitigate interference resulting in higher signal to interference plus noise ratio. Reconfigurable antennas are implemented for various wireless applications that involve cognition and continuous adaptation to the environment as in cognitive radio and Multiple Input Multiple Output (MIMO) systems. Most of the advanced wireless communication applications require highly efficient software controlled dynamic antennas [1-3].

A reconfigurable antenna relies on the electronic switching components like RF MEMS (Radio Frequency Micro Electro Mechanical Systems), Pin diodes or varactors for reconfiguration. The integration of the switches in the antenna structure makes it easier to achieve re-configurability. The effect of environmental conditions on switches are studied and discussed in the past. Thereby, it is evident that environmental conditions have a vital impact on the performance of a switch and thus designing of switches is done taking into consideration the various studies held before.

In [4], to avoid mismatch due to the use of discrete switching elements RF capacitive switch was monolithically integrated with the reconfigurable antenna. In [5] RF MEMS switches are used to connect the different parts of fractal antenna to cover several frequency bands. In $[6,7]$ an air bridged RF MEMS switch in SPST (Single Pole Single Throw) configuration is proposed. In [8,9] the problems of integrating RF MEMS switches into reconfigurable antenna is discussed. In [10], the reliability of the RF MEMS switch is discussed which is affected by the carbon contaminants. The poor reliability of the RF MEMS switches is the

Raji George, rajigeorge2006@gmail.com | 1 DIAT, Pune, India. ${ }^{2}$ SPPU, Pune, India. ${ }^{3}$ R\&DE, Pune, India.

SN Applied Sciences (2019) 1:1087 | https://doi.org/10.1007/s42452-019-1131-y 
limitation for its application in areas like military as well as communication. These antennas can be reconfigured using software. This makes it highly reliable to achieve the required function on user's request. Shannon and Moore had first addressed the fundamentals of improving the reliability [11]. In [12] the mathematics for the fault tolerant circuit of the switching network is discussed. In [13] is a study on a complexity reduction approach after reducing the number of switches.

Graph theory is used for modeling of the antenna structure. This reduces the complexity of analysis. Equivalent configurations can be formulated using graph theory. The use of graph models to represent re-configurable antennas and enhance their functionality by transforming antennas from bulky devices into mathematical and software accessible models. It is observed that the fault tolerance algorithm can be formulated for larger antennas so that the reliability of the reconfigurable antenna can be increased without compromising on the radiation properties of the antenna.

This paper discusses a fault tolerance method for a Frequency reconfigurable pixel antenna proposed in [14]. This paper discusses a grid of $3 \times 3$ pixels. Each pixel is connected to adjacent pixel using RF MEMS switches. Graphs are often used in reconfigurable antenna as a modeling tool. This method improves the reliability by reducing failures. The reconfiguration technique and the environment cause failures. The failure reduction techniques are simulated and verified for the proposed design. The desired radiation property is achieved by detecting the faulty switch and redirecting the current distribution over the antenna surface to achieve the same radiation properties. In [13] is a study on a complexity reduction approach after reducing the number of switches. This technique can also be used to optimize the reconfigurable antenna structure by removing the redundant parts. This reduces the cost and losses. In Sect. 2, the proposed frequency reconfigurable Pixel antenna is discussed with its simulated results in HFSS. Section 3 discusses about fault tolerance method, Sect. 4 discusses the graph modeling of the Reconfigurable Pixel Antenna, Sect. 5 the algorithm for fault tolerance and Sect. 6 the discussion on the results.

\section{Frequency reconfigurable $3 \times 3$ pixel antenna design}

In [14], the frequency reconfigurable antenna has $3 \times 3$ metallic grids with RF MEMS switches between the adjacent rows of patches.

The pixels in the adjacent rows are connected using switches as depicted in Fig. 1. The reconfigurable antenna

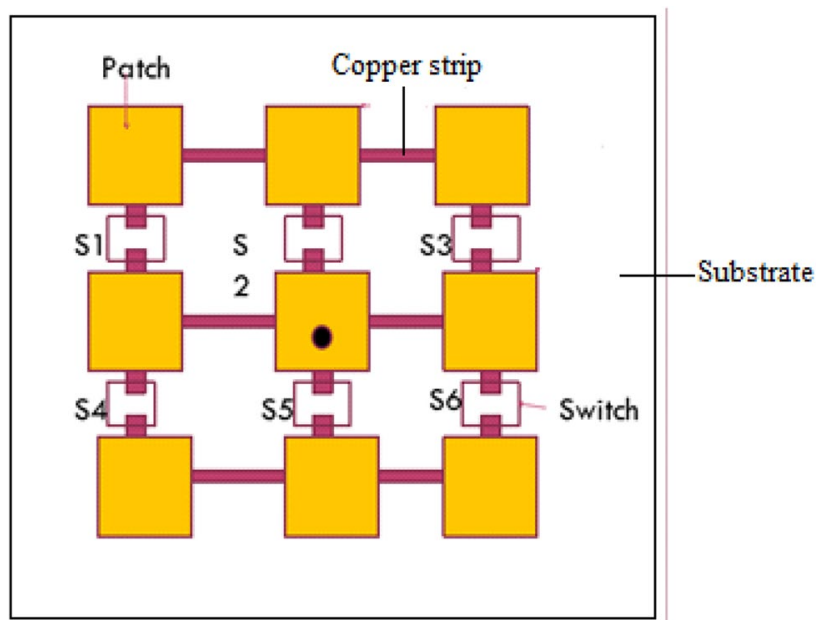

Fig. 1 Design of pixel antenna

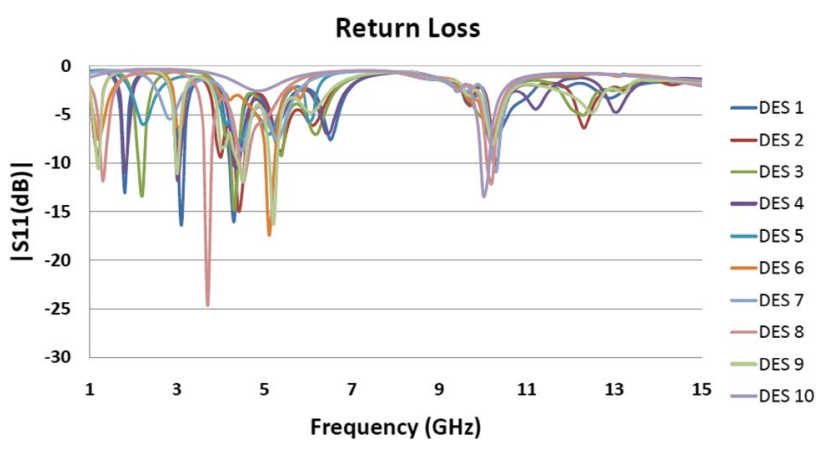

Fig. 2 Simulation result

had 9 pixels interconnected using copper strips and switches. The switches are placed between adjacent rows.

\section{Simulation results in HFSS}

The performance of the Pixel Antenna is simulated in HFSS. Figure 2 shows the simulation result for the different configurations.

Table 1 shows the different configurations with all the possible switch status.

\section{Fabricated reconfigurable pixel antenna}

The frequency reconfigurable antenna is implemented on FR4 substrate with a dielectric constant of 4.4 and loss tangent of 0.02 . The ON/OFF status of the switches provide different configurations. The fabricated frequency reconfigurable antenna is as shown in Fig. $3 a$. 

for the different designs
Table 1 Status of the switches

\begin{tabular}{ll}
\hline Design & Switch status \\
\hline Des 1 & S1, S2 OFF \\
Des 2 & S5, S6 OFF \\
Des 3 & S1 OFF \\
Des 4 & S3 OFF \\
Des 5 & S2 OFF \\
Des 6 & S5 OFF \\
Des 7 & S1, S2, S3, S5 OFF \\
Des 8 & All switch ON \\
Des 9 & S1, S2, S3 OFF \\
Des 10 & All switch OFF \\
\hline
\end{tabular}

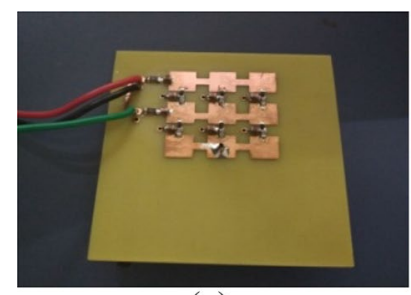

(a)

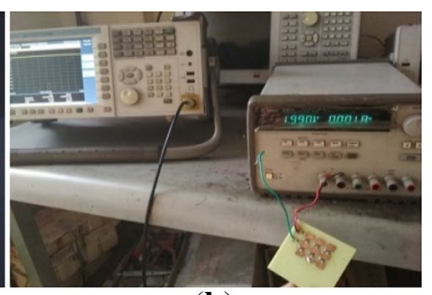

(b)
Fig. 3 a Pixel antenna with PIN diodes. b Measurement using VNA

The frequency re-configurability is achieved by changing the ON/OFF status of the switches. The proposed algorithm is applied for fault tolerance of pixel antenna. In case of a fault in any of the PIN diode switches the proposed fault tolerance algorithm is implemented to achieve the desired frequency.

The Pixel reconfigurable antenna is tested using VNA. Figure $3 \mathrm{~b}$ shows the test setup of a Pixel antenna for measurement on VNA.

The measurements results are shown as Fig. $4 a-c$. The S11 parameters of the three configurations DES 8-DES 10 are shown as Fig. $4 a-c$.

The measured results show that the pixel antenna is frequency reconfigurable with the different status of the switches.

\section{Graph modeling}

A graph can be defined 'as a collection of vertex connected to each other using lines. These lines are called edges. A simple labeled graph over an alphabet $\Sigma$ is represented by $G=(V, E)$ where $V$ is a set of vertices and $E$ is a set of pairs or edges from V. In this paper we are studying undirected graphs where vertices may represent physical entities and edges. These entities and edges in the graph represent the presence of a function resulting from connecting these entities. In the graph for the antenna design

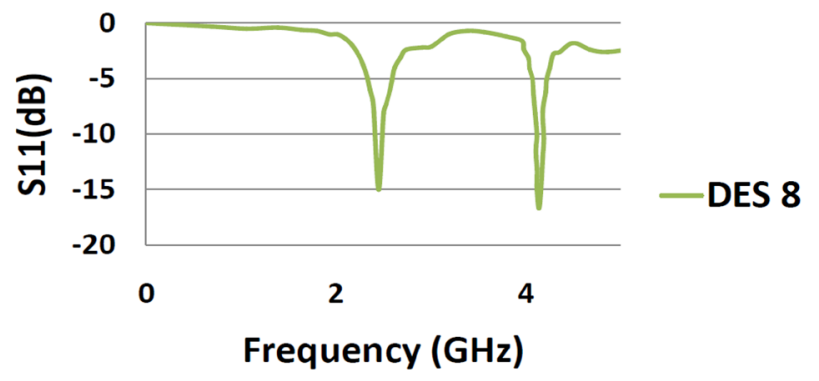

(a) DES 8: When all switches are $O N$

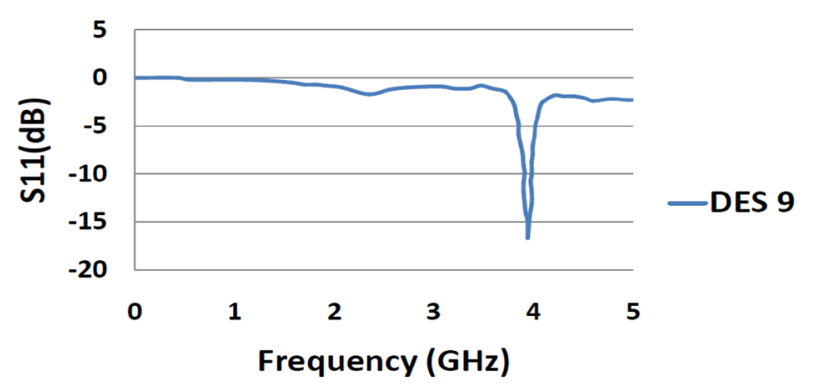

(b) DES 9: When $S 1, S 2, S 3 \mathrm{OFF}$

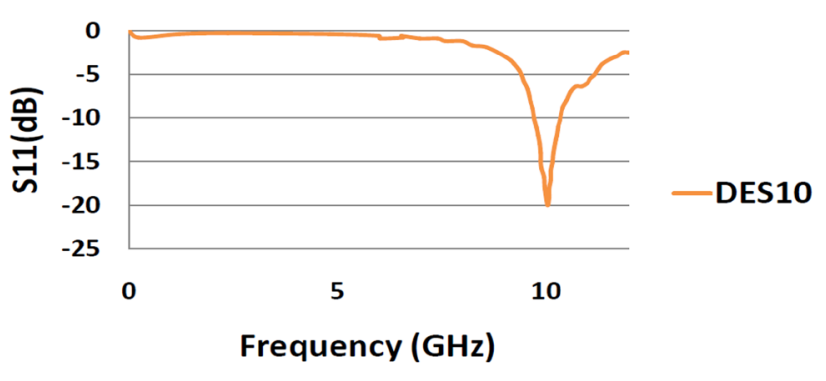

(c) DES 10: When All switches are OFF

Fig. 4 Measured results for the three configurations measured on VNA

between two vertices, an edge is created that forms a connection in the antenna for the specified resonant frequency. Edges are assigned weights. These weights represent the cost or the benefit that needs to be maximized or minimized.

Antenna resonance is directly related to the ON or OFF condition of the switch connecting different parts of the antenna. The flow of the current through these switches leads to different antenna resonances which are expressed through varied graphical interpretations, depending on how we define the vertices, edges and application of these connections.

Graph modeling has been used as a tool for analyzing reconfigurable antenna $[15,16]$. A $3 \times 3$ reconfigurable pixel antenna shown in Fig. 1 can be modeled by the graph as shown in the Fig. 5.

In the Fig. 5 the vertices represent the antenna patches and the edges the connection between them activated 


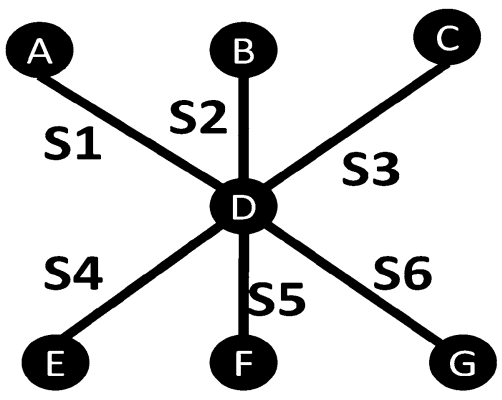

Fig. 5 Graph for the proposed $3 \times 3$ reconfigurable pixel antenna

by the switches. The edges are assigned weights that represent the cost that needs to be maximized or minimized. Algorithms are used for the control and automation of the switching network [17]. Using graph there implementation of the cognitive radio could be made faster and better [18]. The graphs improve the antenna cost and reduce losses.

Different antenna configurations are represented by graph models. There are different configurations that give the same antenna frequency without affecting the other radiation properties. Equivalent configurations are obtained by simulation. A backup configuration can be formed by these equivalent configurations. The same antenna performance can be maintained at a certain frequency. The antenna structure with the RF MEMS switches are shown in Fig. 2. The graph model resonates at $10 \mathrm{GHz}$ for all the configurations and at $4.3 \mathrm{GHz}$ for five different switch configurations. Thus $10 \mathrm{GHz}$ is the most reliable frequency that can be achieved. All the different possible configurations are shown in the Table 1 and the comparative S11 plots are shown in the Fig. 2. These configurations allow to achieve the desired frequency. Table 2 shows all possible configurations for all desired frequencies.

An Alternate method to improve reliability and dependability of the reconfigurable antenna system is to consider the antenna redundant configuration that produces the same electromagnetic behavior for any switch configuration. This redundant configuration should produce a frequency of highest priority. This frequency for this design is $10 \mathrm{GHz}$. It has nine configurations including the no switch configuration. This frequency is the highest priority frequency that can be achieved even in the worst possible scenario where all the switches are faulty.

\section{Fault tolerance}

The incorporation of the switches in the antenna structure increases the complexity and reduces the reliability. Reliability of reconfigurable antenna is a very important factor. Several studies have been conducted in the use
Table 2 Graphs for different configurations

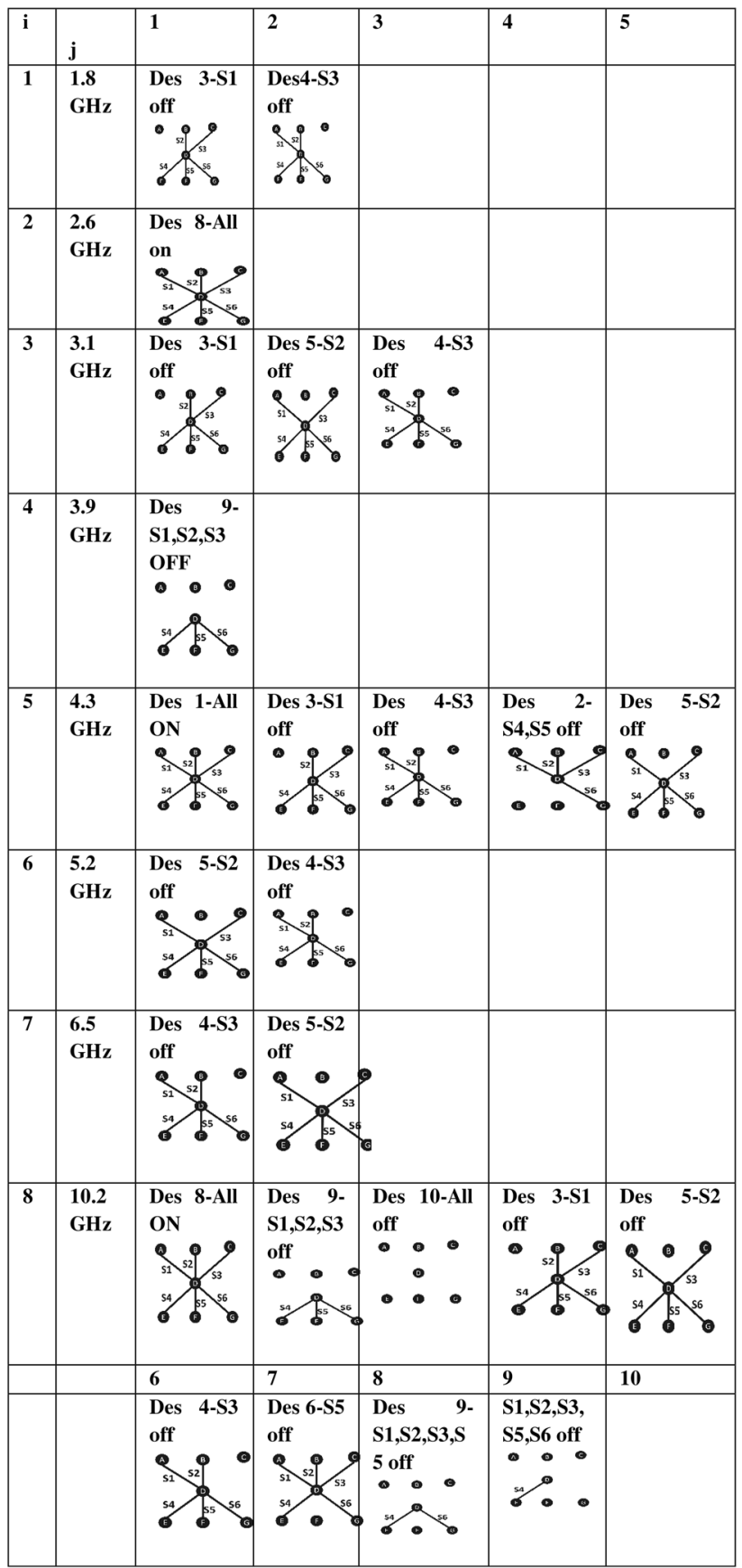

of RF MEMS switches for re-configurability $[4,6,7]$. The improvement of the system reliability was first addressed by Shannon and Moore [11]. They report in their paper that reliability can be increased by reducing redundancy. In [9] fault tolerance circuit switching is discussed switching is discussed to improve reliability. Graph modeling is used for analyzing reconfigurable antennas $[16,17,19]$.

An ideal system never fails which is impossible to achieve in practice. Systems can fail due to different reasons at different levels. Fault tolerance design enables

\section{SN Applied Sciences}


the system to continue its intended operation even when some part or subset of the process becomes faulty. Thus, a fault tolerant system masks failures and the system continues operating $[20,21]$. The fault tolerant system is developed using Graph Modeling of the reconfigurable Pixel Antenna

According to Shannon and Moore's terminology [11], a switch failure occurs when an OFF switch fails to turn ON when requested or when an ON switch fails to turn OFF on request.

The reliability of the antenna gets affected by the switching failure of the switch. The switch failures could be due to various reasons like environment, aging, corrosion and frequency of operation. Using graph theory reliability can be evaluated from the different antenna configurations.

The reliability of the reconfigurable antenna is dependent on the antenna configuration possible for a particular resonant frequency. It is also dependent on the probability to achieve these configurations. The reliability is also stated as inversely proportional to the number of edges needed to create these configurations.

The aim of the methodology is to yield the required antenna frequency with the other entire radiation pattern unaffected. These configurations are called the equivalent configurations. Equivalent configurations can be obtained by simulation of the reconfigurable antenna structure in HFSS. These equivalent configurations form backup configurations to obtain the required frequency maintaining the same antenna performance. Graph models are used to represent equivalent configurations for an antenna structure at a given frequency.

\section{Algorithm for fault tolerance}

During a switch failure, the lost resonance can be restored by using the following proposed methodology. In this methodology the defected switch is identified. Changes are made in the configuration to restore the desired frequency based on the equivalent configuration. For this approach, the designer has to create a library. The library should contain all the possible configurations for all the desired frequencies. To overcome a switch failure and thus restore a lost resonance, the following methodology is proposed. This methodology identifies the defected switch, specifies the desired frequency and changes the antenna topology to restore the desired resonance based on the equivalent configuration. Before applying this approach, the designer creates a library similar to Table 2 in which all possible configurations for all desired frequencies are identified. Each configuration is represented with a graph. RF switches are incorporated into the antenna structure for re-configurability. Due to the failure of a switch the desired frequency is not achieved the following algorithm is applied for the fault tolerance (as shown in Fig. 6).

The following algorithm is followed for fault tolerance: Step 1 Identify the desired frequency.

Step 2 In the library table, a pointer is created at row $\mathrm{i}$ which corresponds to the desired frequency.

Step 3 In case of a faulty operation and the configuration will not be resonating at the desired frequency.

Step $4 \mathrm{~A}$ pointer is created at column $\mathrm{j}$ in the library table, which corresponds to the different switch configurations.

Step 5 Assign the pointer at $\mathrm{j}=1$ for the required row $\mathrm{i}$.

Step 6 Use the configuration at $(i, j)$ and check the desired frequency.

Step 7 If the desired frequency not achieved move to $\mathrm{j}+1$.

Step 8 If $\mathrm{j}<\mathrm{m}$ repeat step 6 and step 7 .

Step 9 If no solution found the desired frequency is unachievable.

\section{Reliability}

From the Table 2 it is observed that for each resonant frequency given in column $i$, there is $m_{i}$ number of possible configurations. Thus Reliability factor $\mathrm{R}$ is directly proportional to the number of maximum possible configurations $\left(m_{i}\right)$ for each row $i$. Table 2 shows that the antenna resonates at $10.2 \mathrm{GHz}$ at nine different switch configurations.

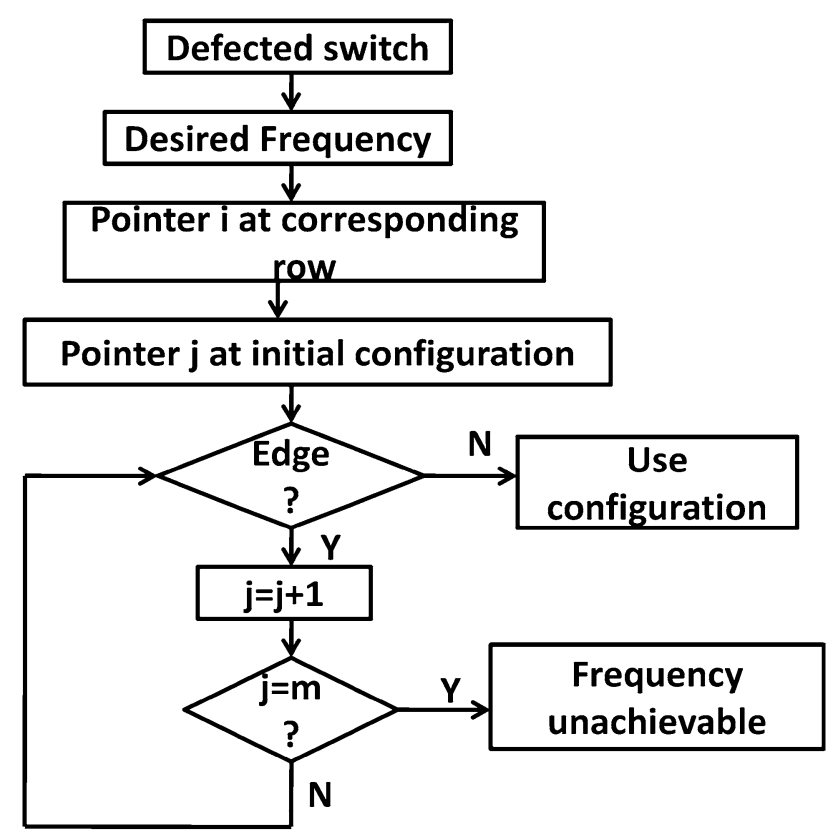

Fig. 6 Flowchart for fault tolerance algorithm 
Reliability Graph

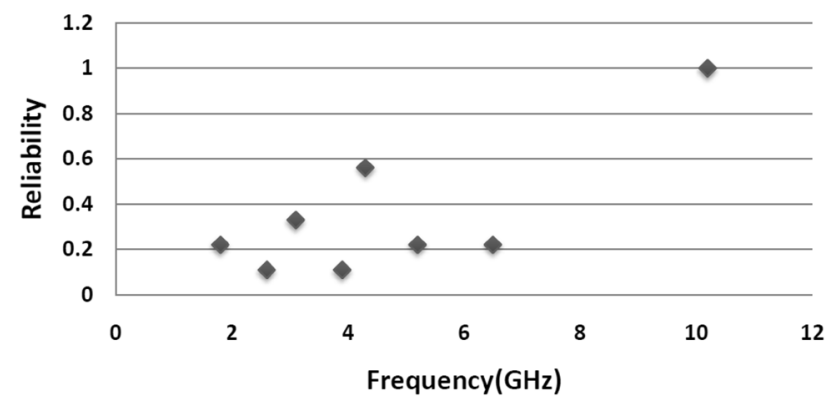

Fig. 7 Reliability graph

This is the No switch configuration when the antenna resonates at $10.2 \mathrm{GHz}$ even when no switches are $\mathrm{ON}$ or else all switches are faulty.

Thus Reliability is defined as the ratio of possible equivalent configurations for a resonant frequency to the maximum possible configurations for the switch circuit. Figure 7 shows the reliability graph.

The reliability graph shows that in the $3 \times 3$ pixel antenna structure, $10.2 \mathrm{GHz}$ is the most reliable frequency and $2.6 \mathrm{GHz}$ and $3.9 \mathrm{GHz}$ are the least reliable frequencies.

\section{Conclusion}

Graph models have been used to model the reconfigurable antenna. The reliability and complexity of such a reconfigurable antenna system is analyzed. An algorithm is written for the fault tolerance of the reconfigurable antenna. This increases the reliability and dependability of the reconfigurable antenna. The desired operating frequency is achieved inspite of fault in the switches using the proposed algorithm.

\section{Compliance with ethical standards}

Conflict of interest On behalf of all authors, the corresponding author states that there is no conflict of interest.

\section{References}

1. Lin W, Wong $\mathrm{H}$ (2016) Polarization reconfigurable aperture-fed patch antenna and array. IEEE Access 4:1510-1517

2. Chung KL, Xie S, Li Y, Liu R, Ji S, Zhang C (2018) A circular polarization reconfigurable Meng-shaped patch antenna. IEEE Access 6:51419-51428

3. Yang G-W, Li J, Cao B, Wei D, Zhou S-G, Deng J (2019) A compact reconfigurable microstrip antenna with multidirectional beam and multipolarization. IEEE Trans Antennas Propag 67(2):1358-1363

SN Applied Sciences
4. Chang H, Quian J, Cetiner BA, De Flaviis F, Bachman M, Li GP (2003) RF MEMS switches fabricated on microwave-laminate printed circuit boards. IEEE Electron Device Lett 24(4):227-229

5. Kingsley N, Anagnostou DE, Tentzeris M, Papapolymerou J (2007) RF MEMS sequentially reconfigurable sierpinski antenna on a flexible organic substrate with novel DC-biasing technique. J Micro Electro Mech Syst 16(5):1185-1192

6. Jung CW, De Flaviis F (2005) RF-MEMS capacitive series of CPW\&MSL configurations for reconfigurable antenna application. In: IEEE antennas and propagation society international symposium. vol 2A, pp 425-428

7. Wang G, Polley T, Hunt A, Papapolymerou J (2005) A high performance tunable RF MEMS switch using barium strontium titanate (BST) dielectrics for reconfigurable antennas and phased arrays. IEEE Antennas Wirel Propag Lett 4:217-220

8. Huff GH, Bernhard JT (2006) Integration of packaged RF MEMS switches with radiation pattern reconfigurable square spiral microstrip antennas. IEEE Trans Antennas Propag 54(2):464-469

9. Anagnostou DE, Zheng G, Chryssomallis M, Lyke J, Ponchak G, Papapolymerou J, Christodoulou CG (2006) Design, fabrication and measurements of an RF-MEMS-based self-similar reconfigurable antenna. IEEE Trans Antennas Propag 54(2):422-432

10. Carton A, Christodoulou CG, Dyck C, Nordquist C (2006) Investigating the impact of carbon contamination on RF MEMS reliability. In: IEEE antennas and propagation international symposium. pp 193-196

11. Moore EF, Shannon CE (1956) Reliable circuits using less reliable relays (Part I and Part II). J Frankl Inst 262(3):191-208 (Sept. 1956); No. 4:pp 281-297 (Oct. 1956)

12. Pippenger N, Lin G (1994) Fault-tolerant circuit-switching networks. SIAM J Discrete Math 7(1):108-118

13. Costantine J (2009) Design, optimization and analysis of reconfigurable antennas. Ph.D thesis, University of New Mexico, Department of Electrical and Computer Engineering

14. George R, Kumar CRS, Gangal SA (2016) Design of a frequency reconfigurable pixel patch antenna for cognitive radio applications. ICCSP, pp 1684-1688

15. Costantine J, Al-Saffar S, Christodoulou CG, Abdallah CT (2011) Reducing redundancies in reconfigurable antenna structures using graph models. IEEE Trans Antennas Propag 59(3):793-801

16. Costantine J, Christodoulou CG (2009) Analyzing reconfigurable antenna structure redundancy using graph models. In: IEEE antennas and propagation society international symposium. pp $1-4$

17. Costantine J, Christodoulou CG, Abdallah CT, Barbin SE (2009) Optimization and complexity reduction of switch-reconfigurable antennas using graph models. IEEE Antennas Wireless Propag Lett 8:1072-1075

18. Tawk Y, Christodoulou CG (2009) A new reconfigurable antenna design for cognitive radio. IEEE Antennas Wireless Propag Lett 8:1378-1381

19. Costantine J, Tawk Y, Christodoulou CG, Abdallah CT (2010) Reducing complexity and improving the reliability of frequency reconfigurable antennas. In: 2010 proceedings of the fourth European conference on antennas and propagation. pp 1-4

20. Avizienis A (1971) Faulty-tolerant computing: an overview. Computer $4(1): 5-8$

21. Kuhl JG, Reddy SM (1995) Fault-diagnosis in fully distributed systems. In: Twenty-fifth international symposium on fault-tolerant computing, 1995, highlights from twenty-five years. p 306

Publisher's Note Springer Nature remains neutral with regard to jurisdictional claims in published maps and institutional affiliations. 